\title{
Adding Epoetin Alfa to Intense Dose-Dense Adjuvant Chemotherapy for Breast Cancer: Randomized Clinical Trial
}

\author{
Volker Moebus, Christian Jackisch, Andreas Schneeweiss, Jens Huober, Hans-Joachim Lueck, Andreas du Bois, \\ Christoph Thomssen, Christian Kurbacher, Walther Kuhn, Ulrike Nitz, Ingo B. Runnebaum, Axel Hinke, Rolf Kreienberg, \\ Michael Untch; on behalf of the AGO Breast Study Group
}

Manuscript received June 12, 2012; revised May 6, 2013; accepted May 13, 2013.

Correspondence to: Volker Moebus, MD, Department of Gynecology and Obstetrics, Klinikum Frankfurt Höchst, Gotenstrasse 6-8, D-65929 Frankfurt, Germany (e-mail: volker.moebus@klinikumfrankfurt.de).

Background

Results

Conclusions

The AGO-ETC trial compared 5-year relapse-free survival of intense dose-dense (IDD) sequential chemotherapy with epirubicin (E), paclitaxel (T), and cyclophosphamide (C) (IDD-ETC) every 2 weeks vs conventional scheduled epirubicin/cyclophosphamide followed by paclitaxel $(E C \rightarrow T)$ (every 3 weeks) as adjuvant treatment in high-risk breast cancer patients. The objective of this study was to evaluate the safety and efficacy of epoetin alfa in a second randomization of the intense dose-dense arm.

One thousand two hundred eighty-four patients were enrolled; 658 patients were randomly assigned to the IDD-ETC treatment group. Within the IDD-ETC group, 324 patients were further randomly assigned to the epoetin alfa group, and 319 were randomly assigned to the non-erythropoiesis-stimulating agent (ESA) control group. Primary efficacy endpoints included change in hemoglobin level from baseline to Cycle 9 and the percentage of subjects requiring red blood cell transfusion. Relapse-free survival, overall survival, and intramammary relapse were secondary endpoints estimated with Kaplan-Meier and Cox regression methods. Except for the primary hypothesis, all statistical tests were two-sided.

Epoetin alfa avoided the decrease in hemoglobin level (no decrease in the epoetin alfa group vs $-2.20 \mathrm{~g} / \mathrm{dL}$ change for the control group; $P<.001$ ) and statistically significantly reduced the percentage of subjects requiring red blood cell transfusion (12.8\% vs $28.1 \% ; P<.0001)$. The incidence of thrombotic events was $7 \%$ in the epoetin alfa arm vs $3 \%$ in the control arm. After a median follow-up of 62 months, epoetin alfa treatment did not affect overall survival, relapse-free survival, or intramammary relapse.

Epoetin alfa resulted in improved hemoglobin levels and decreased transfusions without an impact on relapsefree or overall survival. However, epoetin alfa had an adverse effect, resulting in increased thrombosis.

J Natl Cancer Inst;2013;105:1018-1026

Anemia is frequent in cancer patients, especially in those receiving chemotherapy or radiotherapy and has a negative impact on the patient's quality of life.

Established adjuvant chemotherapeutic regimens of breast cancer patients lead to a clinically significant degree of anemia. Even a standard anthracycline-containing regimen such as the French FEC regimen (5-fluorouracil, epirubicin, cyclophosphamide) induces anemia grades 1 to 3 in $42.4 \%$ of patients (1). Dose-dense combination regimens with granulocyte colony-stimulating factor (G-CSF) support (2) require red blood cell (RBC) transfusions in $13 \%$ of the patients and intense dose-dense regimens such as intense dose-dense (IDD) sequential chemotherapy with epirubicin (E), paclitaxel (T), and cyclophosphamide (C) (IDD-ETC) or doxorubicin (A), paclitaxel (T), and cyclophosphamide (C) (IDDATC) require RBC transfusions in $25 \%$ to $67 \%$ of patients $(3,4)$.

The erythropoiesis-stimulating agent (ESA) epoetin alfa had shown efficacy in treatment $(5-9)$ and prevention $(10,11)$ of chemotherapy-induced anemia in breast cancer patients. However, individual studies (12) and meta-analyses reported an increased risk of death and serious adverse events. Whereas three meta-analyses have indicated an increased risk of mortality with the use of ESAs (13-15), two other meta-analyses did not indicate that ESA use statistically significantly affected disease progression or mortality $(16,17)$. In addition, there is strong evidence from meta-analyses of randomized trials that therapy with epoetin and darbepoetin increases the risk of thromboembolic events $(13,15-17)$.

As a consequence, the revised US Food and Drug Administration label states that ESAs should only be used to treat chemotherapyinduced anemia (18) and they should not be used in malignancies such as early-stage breast cancer when the anticipated treatment outcome is cure.

In contrast with these recommendations, which were not defined at the start of our trial, we evaluated epoetin alfa for prevention of chemotherapy-induced anemia in a curative indication. In a 
preceding phase I/II dose-escalation study with 102 patients, $26 \%$ of these patients required $\mathrm{RBC}$ transfusions (3). This high percentage of $\mathrm{RBC}$ transfusion is not acceptable in the adjuvant setting and was the rationale for performing a second randomization of epoetin alfa vs control in the intense dose-dense ETC arm only. Comparing the effectiveness of IDD-ETC and conventionally scheduled epirubicin/ cyclophosphamide followed by paclitaxel, the 5-year event free survival rates (70\% vs $62 \%)$ and overall survival rates ( $82 \%$ vs $77 \%)$ were statistically significantly improved by IDD-ETC (19).

We report data from the second randomization of epoetin alfa vs control in the IDD-ETC arm. Results of our study will contribute to the still ongoing discussion about the benefit and safety of ESAs in the treatment of cancer patients.

\section{Methods}

\section{Objectives}

The primary objectives were to determine the effect of epoetin alfa treatment compared with non-ESA control for patients in the IDD-ETC group with regard to hemoglobin $(\mathrm{Hb})$ levels during chemotherapy and RBC transfusion requirements.

Secondary objectives were to determine the effect of epoetin alfa treatment with regard to the following: overall and recurrencefree survival after 5 years, intramammary relapse, and assessment of health-related patient-reported outcomes and safety (thrombotic vascular events, serious adverse events, clinical laboratory tests).

The ethics committee of the University of Ulm provided approval for the study. At each participating institution, the study was additionally approved by the local institutional review board. All eligible patients provided written informed consent.

\section{Patients}

Women with histologically confirmed primary breast cancer of stages II to IIIa with four or more tumor-infiltrated axillary lymph nodes were eligible (20). Main inclusion criteria were age between 18 and 65 years, M0 status, and R0 resection of the primary tumor and axilla with a minimum of 10 axillary lymph nodes removed.
Additional eligibility criteria have been previously described in detail (19).

\section{Treatment}

IDD chemotherapy consisted of sequential administration of each of three cycles of epirubicin (E) $\left(150 \mathrm{mg} / \mathrm{m}^{2}\right.$ intravenously as a bolus infusion), paclitaxel (T) $\left(225 \mathrm{mg} / \mathrm{m}^{2}\right.$ intravenously as a 3 -hour infusion), and cyclophosphamide (C) $\left(2500 \mathrm{mg} / \mathrm{m}^{2}\right.$ intravenously as a 2-hour infusion), respectively, every 2 weeks (IDD-ETC; arm A). All patients received filgrastim subcutaneously $(5 \mu \mathrm{g} / \mathrm{kg}$ body weight per day) from days 3 to 10 of each cycle. The standard treatment (arm B) consisted of 4 cycles of epirubicin/cyclophosphamide $\left(90 / 600 \mathrm{mg} / \mathrm{m}^{2}\right)$ followed by 4 cycles of paclitaxel $\left(175 \mathrm{mg} / \mathrm{m}^{2}\right)$ as a 3 -hour infusion $(\mathrm{EC} \rightarrow \mathrm{T})$. All cycles were administered in 3 -week intervals without growth factor support (Figure 1).

Complete blood counts were obtained at each cycle, and $\mathrm{Hb}$ level was measured at least weekly during chemotherapy.

Regular follow-up visits had to be performed every 3 months for the first 3 years, every 6 months during years 4 and 5, and annually thereafter.

\section{Epoetin Alfa Regimen}

Patients in the IDD-ETC arm were randomly assigned to receive epoetin alfa subcutaneously (150 IU/kg three times weekly) or no ESA treatment. ESA treatment started on day 1 and continued for up to 14 days after the last dose of cyclophosphamide. All patients also received $200 \mathrm{mg} /$ day oral iron.

The objective of epoetin alfa treatment was to maintain an appropriate $\mathrm{Hb}$ level of 12.5 to $13.0 \mathrm{~g} / \mathrm{dL}$. Epoetin alfa had to be withdrawn when the $\mathrm{Hb}$ level exceeded $14.0 \mathrm{~g} / \mathrm{dL}$ and could be restarted when the $\mathrm{Hb}$ level dropped to less than $13.0 \mathrm{~g} / \mathrm{dL}$. If the $\mathrm{Hb}$ level dropped by more than $2.0 \mathrm{~g} / \mathrm{dL}$ within a 4 -week period, the dose of epoetin alfa was to be doubled.

Patients with an $\mathrm{Hb}$ level below $9.0 \mathrm{~g} / \mathrm{dL}$ were evaluated for the need of RBC transfusion. The indication for RBC transfusion depended on the symptoms of the patients and was at the discretion of the physician.

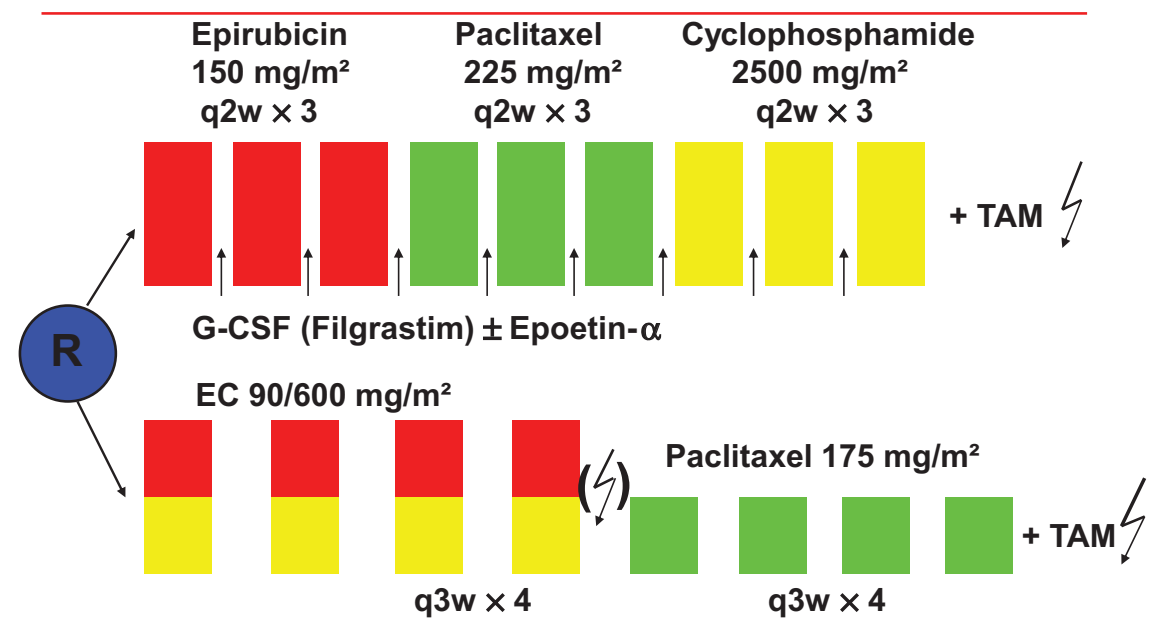

Figure 1. Trial design of AGO trial, intense dose-dense epirubicin, paclitaxel, and cyclophosphamide (IDD-ETC) vs conventionally dosed EC (epirubicin and cyclophosphamide) followed by paclitaxel (T) in patients with four or more lymph nodes. G-CSF = granulocyte colony-stimulating factor; $\mathrm{q} 2 \mathrm{w}=$ every 2 weeks; $\mathrm{q} 3 \mathrm{w}$ = every 3 weeks; TAM =Tamoxifen. 


\section{Statistical Analysis}

Patients were stratified by center, menopausal status (pre- vs postmenopausal), and the number of affected lymph nodes (4-9 vs $\geq 10$ ) at the central fax randomization. Computer-generated lists with permuted blocks of randomly variable size were used. Group assignment in all analyses was based on the randomization result.

The sample size of the Arbeitsgemeinschaft Gynäkologische Onkologie (AGO) trial was mainly driven by the requirements of the main research question on chemotherapy density and its long-term survival-type endpoint. Prospective power calculations revealed that the resulting sample size was sufficient to detect any meaningful difference in $\mathrm{Hb}$ levels and proportions needing transfusion. In addition, the planned patient number attained approximately $85 \%$ power to detect a $10 \%$ difference in the 5-year relapse-free survival rate after a median follow-up of 5 years using a log-rank test.

The numbers of patients who received at least one on-study RBC transfusion were compared between the two groups using Fisher exact test. On-study was defined as the period from randomization to the date of the last cycle of chemotherapy plus 14 days or the date of withdrawal, whichever occurred first.

$\mathrm{Hb}$ values at baseline, change from baseline to each post baseline time point, and change from baseline to the last onstudy assessment were to be presented. Comparison of $\mathrm{Hb}$ levels between the two groups was evaluated with analysis of variance and Wilcoxon tests.

Kaplan-Meier estimates of the relapse-free survival rate through the clinical cutoff date were presented by treatment group for the intent-to-treat population. Comparison of relapse-free survival between the 2 treatment groups was performed using a 2 -sided logrank test with and without the stratification factors for menopausal status and number of positive lymph nodes. The reported $P$ values result from the unstratified analyses. The results from the stratified analyses are virtually identical and therefore not represented.

In addition, Cox regression models, with and without adjustment for the stratification factors, were performed to calculate the hazard ratios (HRs) and associated $95 \%$ confidence intervals (CI). All statistical tests were two-sided, except for the primary endpoint of transfusions for which a one-sided hypothesis was prospectively defined based on previous knowledge of expected differences.

The impact of cancer and its treatment was assessed using the European Organization for Research and Treatment of Cancer Quality of Life Questionnaire, version 3. Patients had to complete the questionnaire before the start of treatment, at every second cycle, at the end of treatment, and at each follow-up visit.

\section{Results}

One thousand two hundred eighty-four patients were recruited between November 1998 and April 2003 from 165 centers in Germany. Six hundred twenty-six subjects were randomly assigned to the $\mathrm{EC} \rightarrow \mathrm{T}$ regimen and 658 subjects were randomly assigned to the IDD-ETC treatment regimen. Of these, 643 subjects were further randomly assigned between the epoetin alfa group $(n=324)$ and the control group $(\mathrm{n}=319)$ (CONSORT diagram, Figure 2). Median follow-up duration was 62 months, but the study is ongoing for continued 10-year follow-up.
The safety population included 627 subjects, 309 subjects in the epoetin alfa group and 318 subjects in the control group. The 16 subjects excluded from the safety population were either assigned to the control group but received ESA treatment ( $\mathrm{n}=1$ subject) or were assigned to the epoetin alfa group but did not receive epoetin alfa ( $\mathrm{n}=15$ subjects). The per-protocol population included 511 subjects, 258 subjects in the epoetin alfa group and 253 in the control group. All of these subjects received nine cycles of chemotherapy. Excluded from the per-protocol population were subjects with unknown baseline Eastern Cooperative Oncology Group/World Health Organization performance status, subjects with less than four positive lymph nodes at baseline, and subjects who did not receive their assigned treatment. The majority of subjects excluded from the per-protocol population failed to complete nine cycles of chemotherapy.

Eighty-three percent of subjects completed the IDD-ETC arm (85\% epoetin alfa group and $81 \%$ control group). "Serious adverse event" was cited as the reason for discontinuation for the majority of subjects from both the epoetin alfa group ( $n=32$ of 49 subjects) and the control group ( $\mathrm{n}=38$ of 60 subjects). The two treatment groups were generally similar with respect to the demographic and baseline characteristics summarized in Table 1.

\section{Epoetin Alfa Dosing}

The median duration of epoetin alfa treatment was 18 weeks (mean $=16.9$ weeks), and the median weekly dose received was 452 $\mathrm{IU} / \mathrm{kg}$ (mean = $441 \mathrm{IU} / \mathrm{kg}$ per week). Although epoetin alfa dosing information had to be reported in the case report form as the number of units administered per kilogram of body weight, a fixed dose of $10000 \mathrm{IU}$ was specified for some subjects. In these instances, a per-kilogram dose was calculated using the subject's body weight.

\section{Hb Levels}

The median baseline Hb level was somewhat lower in the epoetin alfa group $(12.40 \mathrm{~g} / \mathrm{dL}$; interquartile range [IQR] $=11.7-13.3 \mathrm{~g} /$ $\mathrm{dL})$ than the control group $(12.80 \mathrm{~g} / \mathrm{dL}$; IQR $=12.2-13.6 \mathrm{~g} / \mathrm{dL})$ and decreased for both groups over the first three cycles of chemotherapy. However, there was no decline from baseline to cycle 9 in the epoetin alfa group $(12.4 \mathrm{~g} / \mathrm{dL}$ at both cycle 1 and cycle 9$)$. In contrast, the decline from baseline to cycle 9 for patients in the control group was $2.20 \mathrm{~g} / \mathrm{dL}(P<.001)$. Results of this analysis for the intent-to-treat population were similar to those for the per-protocol population, and the results were confirmed when an analysis of variance model was employed.

Figure 3 reflects the myelosuppressive toxicities of IDD-ETC and the effect of epoetin alfa. Each point in Figure 3 represents the mean of the $\mathrm{Hb}$ values measured for a given cycle. The statistically significant difference between treatment groups is reflected in the final separation of $\mathrm{Hb}$ level curves.

\section{Transfusion requirements}

For the intent-to-treat population, more than twice as many subjects in the control group received at least one RBC transfusion during chemotherapy as compared with subjects in the epoetin alfa group (86 [28.1\%] vs 41 [12.8\%] subjects, respectively). The difference between groups was statistically significant $(P<.0001)$. The estimated transfusion odds ratio for the overall treatment 


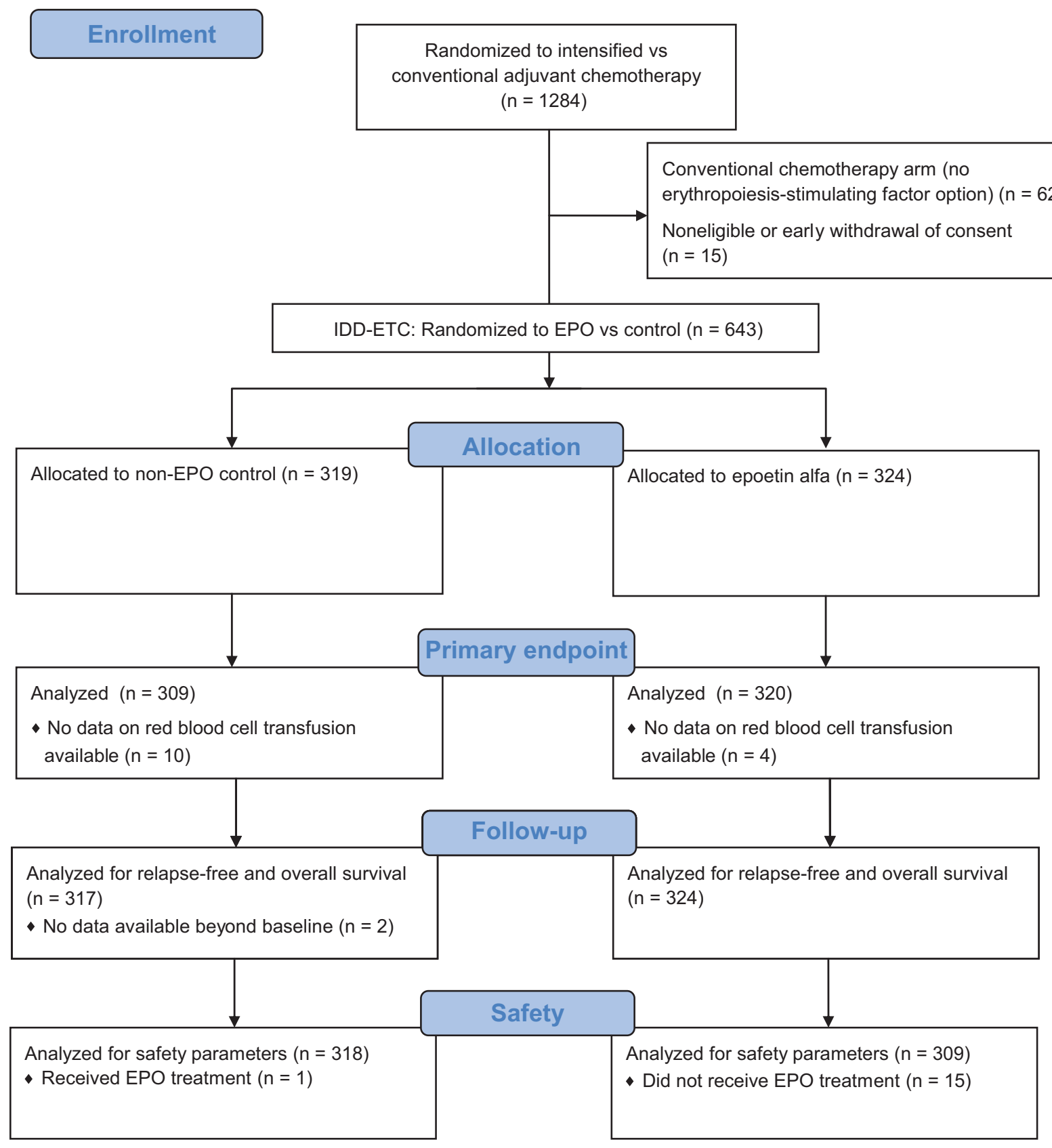

Figure 2. CONSORT trial flow diagram. EPO = epoetin alfa; ESA = erythropoiesis-stimulating agent; IDD-ETC = intense dose-dense sequential chemotherapy with epirubicin, paclitaxel, and cyclophosphamide.

period was $0.37(95 \% \mathrm{CI}=0.25$ to 0.57$)$. Similar results were obtained when the per-protocol population was analyzed.

Most transfusions, regardless of treatment group, occurred during cycles 7 to 9 . However, the number of subjects in the control group who received transfusions tended to increase steadily from cycle 1 onward to cycle 9 , whereas the number of subjects in the epoetin alfa group who received transfusions increased mainly during cycles 7 to 9 .

\section{Relapse-Free Survival}

The Kaplan-Meier estimates of relapse-free survival for the intent-totreat population are shown in Figure 4. This analysis took into account any disease relapse or death as events. The 5 -year relapse free survival rates were $71 \%(95 \% \mathrm{CI}=66 \%$ to $76 \%)$ and $72 \%(95 \% \mathrm{CI}=67 \%$ to $77 \%)$ for subjects in the control and epoetin alfa groups, respectively. The hazard ratio was $1.03(95 \% \mathrm{CI}=0.77$ to 1.37$)$, and the difference between groups was not statistically significant $(P=.86)$.

\section{Overall Survival}

The Kaplan-Meier estimates of overall survival for the intent-totreat population are shown in Figure 5. The hazard ratio was 0.97 ( $95 \% \mathrm{CI}=0.67$ to 1.41 ), and the difference between groups was not statistically significant $(P=.89)$.

The 5 -year overall survival rates were $81 \%$ (95\% CI $=76 \%$ to $86 \%)$ and $83 \%(95 \% \mathrm{CI}=78 \%$ to $87 \%)$ for subjects in the epoetin alfa and control groups, respectively $(P=.89)$. 
Table 1. Demographic and baseline characteristics (intent-to-treat population)*

\begin{tabular}{|c|c|c|}
\hline Characteristic & Non-EPO control, No. (\%) & EPO, No. (\%) \\
\hline No. & 319 & 324 \\
\hline \multicolumn{3}{|l|}{ Age, years } \\
\hline Median & 52 & 50 \\
\hline Range & $28-67$ & $29-65$ \\
\hline \multicolumn{3}{|l|}{ Body mass index, $\mathrm{kg} / \mathrm{m}^{2}$} \\
\hline Median & 24.5 & 24.4 \\
\hline Range & $17-42$ & $17-48$ \\
\hline \multicolumn{3}{|c|}{ Positive lymph nodes, No. (\%) } \\
\hline $4-9$ & $185(58)$ & $191(59)$ \\
\hline$\geq 10$ & $134(42)$ & $133(41)$ \\
\hline \multicolumn{3}{|l|}{ Menopausal status, No. (\%) } \\
\hline Premenopausal & $143(45)$ & $163(51)$ \\
\hline Postmenopausal & $176(55)$ & $160(49)$ \\
\hline \multicolumn{3}{|l|}{ Tumor stage, No. (\%) } \\
\hline pT1 & $100(31)$ & $81(25)$ \\
\hline pT2 & $172(54)$ & $190(59)$ \\
\hline рT3 & $46(14)$ & $50(15)$ \\
\hline \multicolumn{3}{|c|}{ Baseline hemoglobin level, g/dL } \\
\hline No. & 303 & 313 \\
\hline Median & $12.8 \mathrm{~g} / \mathrm{dL}$ & $12.4 \mathrm{~g} / \mathrm{dL}$ \\
\hline Range & $9.0-16.0 \mathrm{~g} / \mathrm{dL}$ & $9.0-16.0 \mathrm{~g} / \mathrm{L}$ \\
\hline \multicolumn{3}{|l|}{ HER2+ } \\
\hline No. & 319 & 322 \\
\hline Positive, No. (\%) & $83(26)$ & $79(25)$ \\
\hline Negative, No. (\%) & $183(57)$ & 189 (59) \\
\hline Not performed, No. (\%) & $53(17)$ & $54(17)$ \\
\hline \multicolumn{3}{|l|}{ ECOG } \\
\hline No. & 312 & 315 \\
\hline ECOG 0, No. (\%) & $260(83)$ & $254(81)$ \\
\hline ECOG 1, No. (\%) & $52(17)$ & $61(19)$ \\
\hline \multicolumn{3}{|l|}{ ER status } \\
\hline No. & 317 & 322 \\
\hline Positive, No. (\%) & $221(70)$ & $244(76)$ \\
\hline Negative, No. (\%) & $96(30)$ & $78(24)$ \\
\hline
\end{tabular}

* ECOG $=$ Eastern Cooperative Oncology Group; $E R=$ estrogen receptor; $E P O=$ epoetin alfa.

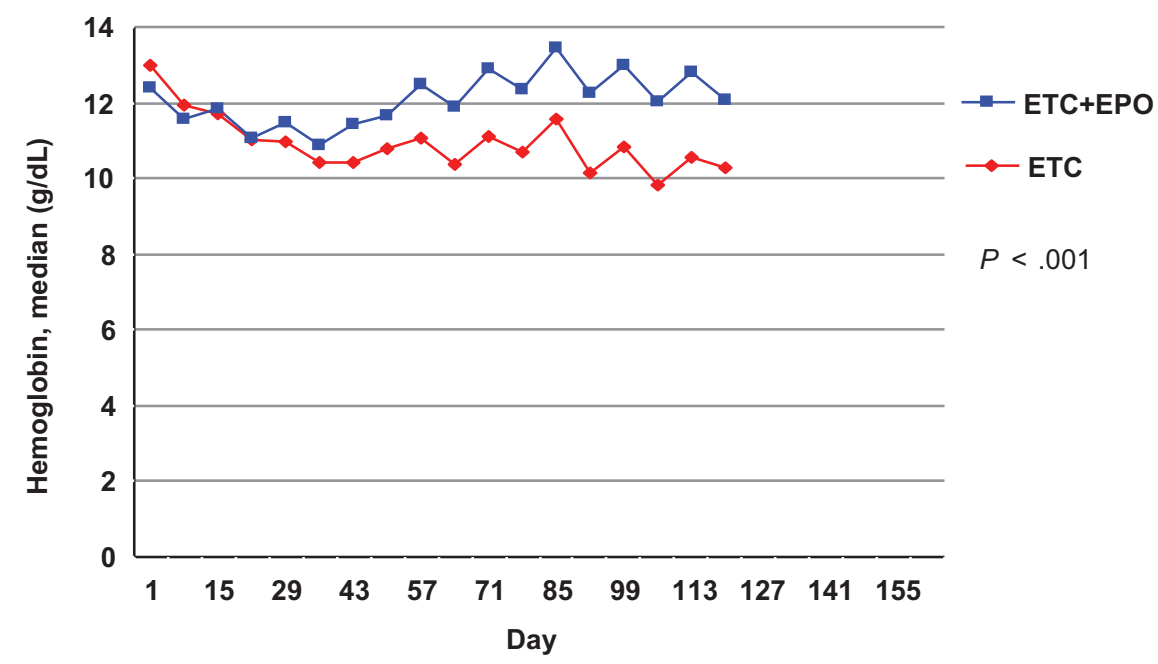

Figure 3. Hemoglobin level ( $\mathrm{g} / \mathrm{dL}$ ) by cycle (Intent-to-treat population). Based on available measurements in the patients receiving the respective chemotherapy cycle. Analysis of variance and Wilcoxon test. EPO = epoetin alfa; ETC = epirubicin, paclitaxel, and cyclophosphamide. 
Time to relapse by therapy

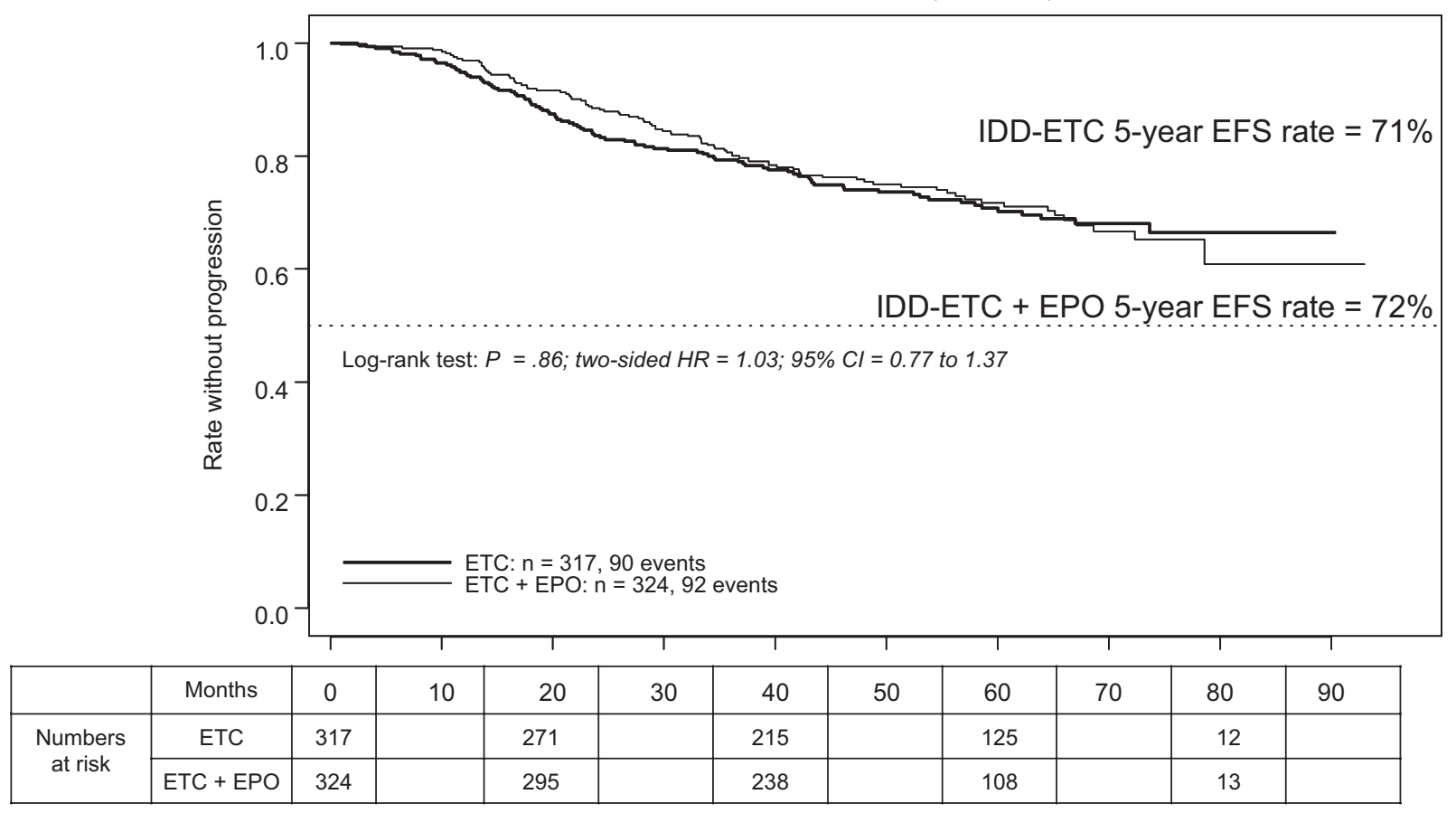

Figure 4. Kaplan-Meier curve of relapse-free survival (intent-to-treat population) Log-rank test. All statistical tests were two-sided. $\mathrm{Cl}=\mathrm{confidence}$ interval; EFS = event-free survival; EPO = epoetin alfa; ETC = epirubicin, paclitaxel, and cyclophosphamide; $\mathrm{HR}=$ hazard ratio.

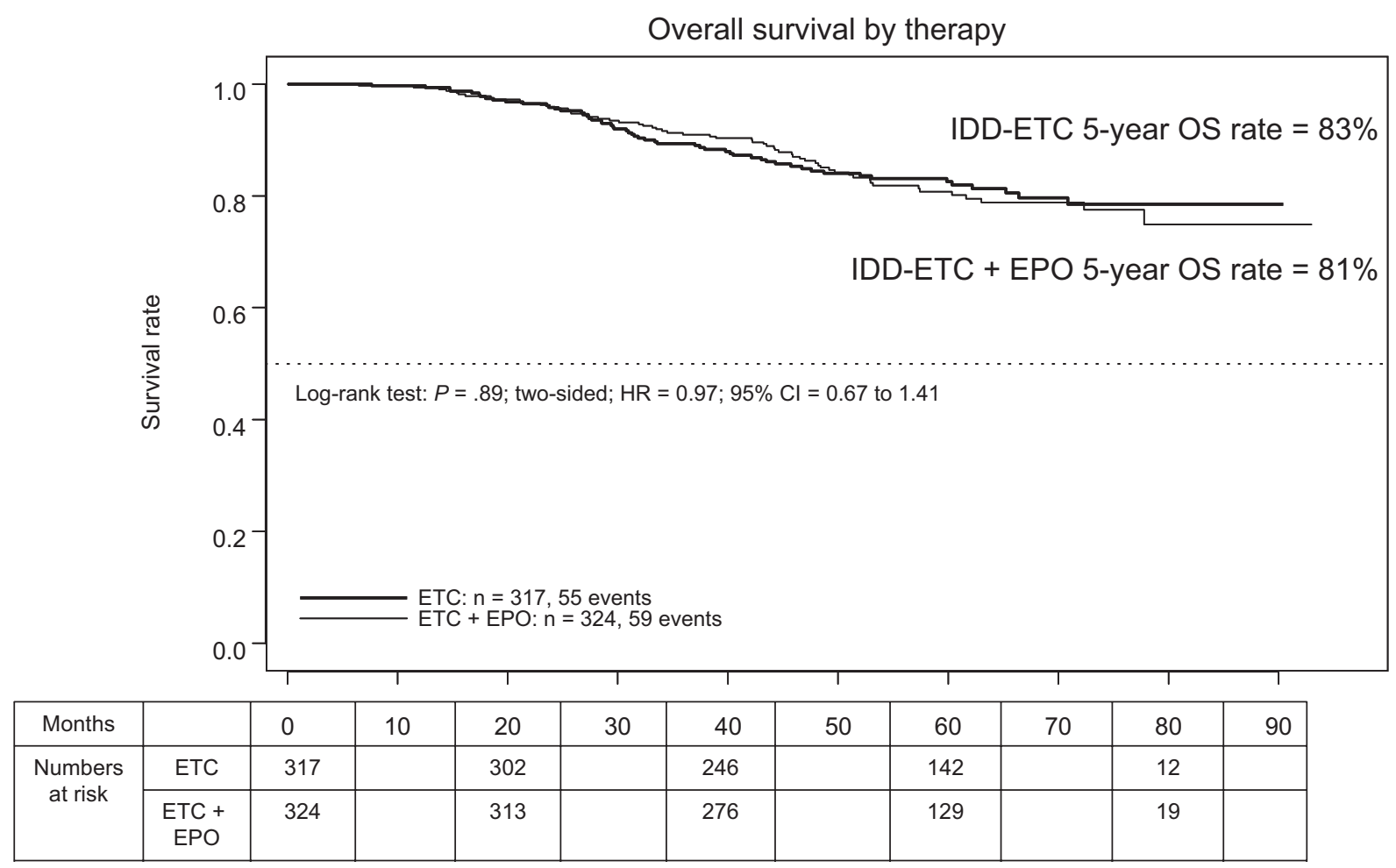

Figure 5. Kaplan-Meier curve of overall survival (intent-to-treat population). Log-rank test. All statistical tests were two-sided. $\mathrm{Cl}=\mathrm{confidence}$ interval; EPO = epoetin alfa; ETC = epirubicin, paclitaxel, and cyclophosphamide; HR = hazard ratio; OS = overall survival.

\section{Safety}

The overall incidence of serious adverse events was $11 \%$ and was similar in both groups: $10 \%$ in the epoetin alfa group and $13 \%$ in the control group.
Thirty-nine $(13 \%)$ of the 309 subjects in the epoetin alfa group and $22(7 \%)$ of the 318 subjects in the non-ESA control group were reported to have experienced at least one thrombotic vascular event while on chemotherapy. The summary of clinically relevant 
Table 2. Clinically relevant thrombotic vascular events (safety population)*

\begin{tabular}{|c|c|c|c|}
\hline $\begin{array}{l}\text { Chosen MedDRA body system/organ } \\
\text { class Chosen MedDRA preferred term }\end{array}$ & $\begin{array}{l}\text { Non-EPO control } \\
(\mathrm{n}=318) \text {, No. }(\%)\end{array}$ & $\begin{array}{c}\text { EPO } \\
(n=309), \text { No. }(\%)\end{array}$ & $\begin{array}{c}\text { Total }(\mathrm{N}=627) \text {, } \\
\text { No. }(\%)\end{array}$ \\
\hline Total No. of subjects with adverse events & $10(3)$ & $22(7)$ & $32(5)$ \\
\hline Vascular disorders & $10(3)$ & $22(7)$ & $32(5)$ \\
\hline Venous thrombosis & 0 & $2(1)$ & $2(<1)$ \\
\hline Arterial thrombosis & 0 & $1(<1)$ & $1(<1)$ \\
\hline Deep vein thrombosis & 0 & $1(<1)$ & $1(<1)$ \\
\hline Respiratory, thoracic, and mediastinal disorders & $1(<1)$ & 0 & $1(<1)$ \\
\hline Pulmonary embolism & $1(<1)$ & 0 & $1(<1)$ \\
\hline
\end{tabular}

* Incidence is based on the number of subjects experiencing at least one adverse event, not the number of events. Percentage calculated with the number of subjects in each group as the denominator. EPO = epoetin alfa; MedDRA = Medical Dictionary for Regulatory Activities.

thrombotic vascular events is presented in Table 2. Fewer subjects in the control group experienced clinically relevant thrombotic vascular events compared with subjects in the epoetin alfa group (10 [3\%] vs 22 [7\%] subjects, respectively; $P=.03$, Fisher exact test).

\section{Quality of Life}

Results for health-related patient-reported outcome analyses are not presented because of the large amount of missing baseline data (in excess of $40 \%$ of baseline measurements were missing).

\section{Discussion}

The use of epoetin alfa in the IDD-ETC arm statistically significantly reduced the rate of RBC transfusion $(12.8 \%$ vs $28.2 \%$ of subjects; $P<.001)$ and avoided the chemotherapy-induced decline in $\mathrm{Hb}$ level as compared with patients in the non-ESA control group.

Regarding the discussions of the last decade, there are concerns that ESAs could increase mortality in cancer patients. The Breast Cancer Erythropoietin Survival Trial (BEST) (12) was one of the first randomized studies that reported an increased mortality in metastatic breast cancer patients receiving epoetin alfa. The first meta-analysis that identified increased mortality by ESA use $(\mathrm{HR}=1.10 ; 95 \% \mathrm{CI}=1.01$ to 1.20$)$ was published by Bennett et al. (13). These authors identified eight studies that individually demonstrated increased mortality and/or tumor progression among patients treated with ESAs (12,21-27). However, it should be noted that only two of these trials treated breast cancer patients (metastatic and neoadjuvant); dominant tumor entities were head and neck, cervical, lympho-proliferative, and non-small cell lung carcinoma. Bohlius et al. (14) reported results from the independent patient data meta-analysis for on-study deaths and overall survival and concluded that ESA use increased mortality during the active study period $(\mathrm{HR}=1.17 ; 95 \% \mathrm{CI}=1.06$ to 1.30$)$ and worsened overall survival $(\mathrm{HR}=1.06 ; 95 \% \mathrm{CI}=1.00$ to 1.12$)$. However, in patients receiving chemotherapy, a statistically significant difference between the ESA and control groups (on-study death $\mathrm{HR}=1.10$, $95 \% \mathrm{CI}=0.98$ to 1.24 ; overall survival $\mathrm{HR}=1.04,95 \% \mathrm{CI}=0.97$ to 1.11 ) was not observed. An erratum regarding a correction of the upper limit of the $x$-axis should be noted (28). The meta-analysis of Tonelli et al. (15) also confirmed that all-cause mortality during treatment was statistically significantly higher in the group receiving erythropoiesis-stimulating therapy than in the control group.
In contrast, two meta-analyses $(16,17)$ could not confirm these safety concerns. The Glaspy et al. meta-analysis (16) included studies from the 2006 Cochrane meta-analysis, studies published/ updated since the 2006 Cochrane report, and unpublished trial data from Amgen and Johnson and Johnson. Their results indicated that ESA use did not statistically significantly affect mortality $(\mathrm{HR}=1.06 ; 95 \% \mathrm{CI}=0.97$ to 1.15$)$ or disease progression $(26$ studies: $\mathrm{HR}=1.01 ; 95 \% \mathrm{CI}=0.90$ to 1.14 ).

Ludwig et al. (17) conducted a pooled analysis of individual patient-level data from all randomized, double-blind, placebo-controlled trials in patients receiving darbepoetin alfa or placebo. They found no association between darbepoetin alfa and risk for death or disease progression. Taken together, the results and conclusions of these meta-analyses remain controversial.

In our analysis of the IDD-ETC arm, the Kaplan-Meier analysis of relapse-free survival showed no difference between the epoetin alfa and the control group. The hazard ratio was $1.03(95 \%$ $\mathrm{CI}=0.77$ to $1.37 ; P=.86$ ). Further, for the overall survival analysis, the Kaplan-Meier analysis showed no significant difference between the two arms $(P=.89)$, neither indicating a benefit of maintaining $\mathrm{Hb}$ levels and reducing transfusion nor showing any hint for a detrimental effect of epoetin application during dosedense and dose-intensified chemotherapy.

Our results are in accordance with two large recently reported studies in the adjuvant treatment of lymph node-positive breast cancer patients receiving chemotherapy. In the previously reported ARAplus trial (29), patients receiving either the TAC (docetaxel, doxorubicin, cyclophosphamide) or the FEC (5-fluorouracil, epirubicin, cyclophosphamide) regimen were randomly assigned between darbepoetin alfa or standard supportive care. The analysis of 1198 evaluable patients showed no difference in the event-free and overall survival between the groups, and no excess on-study mortality was observed in the ESA arm when compared with control patients.

The NSABP B-38 trial compared TAC vs dose-dense AC $\rightarrow$ paclitaxel (every 2 weeks) \pm gemcitabine (30). ESAs were given in all three arms if $\mathrm{Hb}$ was below $11 \mathrm{~g} / \mathrm{dL}$. A statistically significantly higher incidence of grade 2 anemia was reported for the two dosedense arms in comparison with TAC. Two thousand one hundred forty-nine of 4894 included patients in this trial received ESAs. With a median follow-up duration of 5.3 years, exploratory analysis revealed no differences regarding disease-free $(\mathrm{HR}=1.02)$ or overall survival $(\mathrm{HR}=1.04)$. 
The fourth randomized trial in the curative setting of breast cancer patients was the neoadjuvant PREPARE trial $(26,31)$. The PREPARE study compared a sequential IDD regimen of epirubicin and paclitaxel (every 2 weeks) followed by conventional cyclophosphamide, 5-fluorouracil, and methotrexate vs conventionally scheduled epirubicin/ cyclophosphamide followed by paclitaxel. Again, in both arms, subjects were randomly assigned to receive either darbepoetin alfa or no treatment to prevent anemia and potentially augment the therapeutic effects of chemotherapy. Three-year disease-free survival was $74.3 \%$ with darbepoetin vs $80 \%$ without $(\mathrm{HR}=1.31 ; P=.06)$, and overall survival was $88 \%$ with darbepoetin vs $91.8 \%$ without $(\mathrm{HR}=1.33$; $P=.14)$. Although not statistically significant, these results suggest a negative impact on disease progression.

Although all four trials were conducted with curative intent, they differ in important points. Only the PREPARE trial included patients in the neoadjuvant treatment setting. The ETC trial is the only trial that exclusively recruited patients undergoing an anemiacausing regimen. WSG-ARA plus, PREPARE, and NSABP B-38 included, at least in part, patients receiving non-anemia-causing regimens. Correspondingly, the mean $\mathrm{Hb}$ concentration for the epoetin alfa treatment group in the IDD-ETC arm was lower at the end of chemotherapy $(12.4 \mathrm{~g} / \mathrm{dL})$ as compared with patients in the PREPARE study $(13.6 \mathrm{~g} / \mathrm{dL})$.

In a review of the literature, preclinical data are ambiguous regarding a direct or indirect effect of ESAs on tumor growth (32). A study by Bennett et al. (33) reported that academic researchers without pharmaceutical manufacturer research/funding more often report both direct and indirect effects of ESAs on tumor growth, in contrast with researchers with pharmaceutical funding. The results of these studies support the hypothesis that the risk of potential tumor progression and decreased survival by the use of ESAs could be mainly restricted to the metastatic and neoadjuvant treatment situations $(12,31)$ in which patients have relevant tumor load. In this clinical setting, ESAs may accentuate tumor growth by stimulation of erythropoietin receptors on tumor cells (despite the questions on antibody specificity in epoetin (EPO) receptor measurement) $(34,35)$. In contrast, trials in the adjuvant setting, such as AGO-ETC, ARA plus (29), and NSABP B-38 (30), showed no adverse effect of ESAs on disease-free and overall survival.

There is now strong and consistent evidence from individual trials and from meta-analyses that therapy with ESAs increases the risk of thromboembolic events. Bennett et al. (13) reported a 1.57-fold increased venous thromboembolism (VTE) risk with ESA administration, which was confirmed by the majority of randomized phase III trials (36) and published overviews $(13,15-17)$. Specific risk factors in addition to the general risk factors for thrombotic events have not been defined in these trials. Our study also confirmed an elevated risk of venous thrombotic events in patients receiving epoetin alfa, but fortunately we observed no pulmonary embolism or fatal event. This increased risk of thromboembolism is consistent with the current epoetin alfa labeling.

The IDD-ETC regimen is highly effective and safe (19) but has a pronounced hematological toxicity. Our study did not evaluate treatment of chemotherapy-induced anemia as much as prevention of this anemia. With regard to the rapid decline of the $\mathrm{Hb}$ level in the non-ESA control group despite a more than twofold higher transfusion rate, primary prophylaxis of anemia is indicated. This is in contrast with guidelines in Canada and the United States and with the pharmaceutical manufacturers product label that indicate these agents should not be used when patients receive chemotherapy with curative intent. Primary prevention is, at least, partially supported by the updated guidelines of the European Organization for Research and Treatment of Cancer, ${ }^{37}$ which considers ESA use in selected asymptomatic chemotherapy patients to prevent a further decline in Hb level according to individual factors (eg, type/ Intensity and duration of chemotherapy) $(37,38)$.

We have shown a beneficial effect of epoetin alfa in the adjuvant treatment of breast cancer patients receiving IDD chemotherapy without deleterious effects on disease progression or mortality after almost 5 years of follow-up. However, the size of our study was not large enough to detect any negative effects on survival between epoetin alfa and no epoetin alfa. In accordance with the literature, we confirmed the known detrimental effects on thrombotic complications. With the exception of this risk, ESAs appear to be safe drugs for the treatment of chemotherapy-induced anemia or the primary prevention of anemia in patients with IDD chemotherapy regimens. Regarding the effects of ESAs, our results were confirmed by a recent clinical trial whose formal primary endpoint was survival. Data from this study also suggest that ESAs have no detrimental effect on disease progression in breast cancer patients undergoing adjuvant chemotherapy (29).

\section{References}

1. Bonneterre J, Roché H, Kerbrat P, et al. Epirubicin increases long-term survival in adjuvant chemotherapy of patients with poor-prognosis, nodepositive, early breast cancer: 10-year follow-up results of the FrenchAdjuvant Study Group 05 randomized trial. 7 Clin Oncol. 2005;23:2686-2693.

2. Citron ML, Berry DA, Cirrincione C, et al. Randomized trial of dose-dense versus conventionally scheduled and sequential versus concurrent combination chemotherapy as postoperative adjuvant treatment of node-positive primary breast cancer: first report of intergroup trial C9741/Cancer and Leukemia Group B Trial 9741.7 Clin Oncol. 2003;21:1431-1439.

3. Moebus V, Heilmann V, Schneeweiss A, et al. Supportive therapy with epoetin-alpha in breast cancer patients (pts) receiving dose-dense sequential chemotherapy with epirubicin, paclitaxel and cyclophosphamide (ETC). Eur 7 Cancer. 2001;37(Suppl6):S171.

4. Hudis C, Fornier M, Riccio L, et al. 5-year results of dose-intensive sequential adjuvant chemotherapy for woman with high-risk node-positive breast cancer: a phase II study. 7 Clin Oncol. 1999;17:1118-1126.

5. Abels RI. Use of recombinant human erythropoietin in the treatment of anemia in patients who have cancer. Semin Oncol. 1992;19:29-35.

6. Glaspy J, Bukowski R, Steinberg D, et al. Impact of therapy with epoetin alfa on clinical outcomes in patients with nonmyeloid malignancies during cancer chemotherapy in community oncology practice. 7 Clin Oncol. 1997;15:1218-1234.

7. Gabrilove JL, Cleeland CS, Livingston RB, et al. Clinical evaluation of once-weekly dosing of epoietin alfa in chemotherapy patients: improvements in hemoglobin and quality of life are similar to three-times-weekly dosing. 7 Clin Oncol. 2001;19:2875-2882.

8. Littlewood TJ, Bajetta E, Nortier JW, et al. Effects of epoetin alfa on hematologic parameters and quality of life in cancer patients receiving non-platinum chemotherapy: Results of a randomized, double-blind, placebo-controlled trial. 7 Clin Oncol. 2001;19:2865-2874.

9. Shasha D, George MJ, Harrison LB. Once-weekly dosing of epoetinalpha increases hemoglobin and improves quality of life in anemic cancer patients receiving radiation therapy either concomitantly or sequentially with chemotherapy. Cancer. 2003;98:1072-1079.

10. Chang J, Couture F, Young S, et al. Weekly epoetin alfa maintains hemoglobin, improves quality of life, and reduces transfusion in breast cancer patients receiving chemotherapy. 7 Clin Oncol. 2005;23:2597-2605. 
11. Del Mastro L, Venturini M, Lionetto R, et al. Randomized phase III trial evaluating the role of erythropoietin in the prevention of chemotherapyinduced anemia, 7 Clin Oncol. 1997;15:2715-2721.

12. Leyland-Jones B, Semiglazov V, Pawlicki M, et al. Maintaining normal hemoglobin levels with epoetin alfa in mainly nonanemic patients with metastatic breast cancer receiving first-line chemotherapy: a survival study. 7 Clin Oncol. 2005;23:5960-5972.

13. Bennett CL, Silver SM, Djulbegovic B, et al. Venous thromboembolism and mortality associated with recombinant erythropoietin and darbepoetin administration for the treatment of cancer-associated anemia. $7 A M A$. 2008;299:914-924.

14. Bohlius J, Schmidlin K, Brillant C, et al. Recombinant human erythropoiesis stimulating agents and mortality in patients with cancer: a meta-analysis of randomized trials. Lancet. 2009;373:1532-1542.

15. Tonelli M; Hemmalgarn B, Reimann T, et al. Benefits and harms of erythropoiesis-stimulating agents for anemia related to cancer. A meta-analysis. CMA7. 2009;180:E62-E71

16. Glaspy J, Crawford J, Vansteenkiste J, et al. Erythropoiesis-stimulating agents in oncology: a study-level meta-analysis of survival and other safety outcomes. Br 7 Cancer. 2010;102:301-315.

17. Ludwig H, Crawford J, Osterborg A, et al. Pooled analysis of individual patient-level data from all randomized, double-blind, placebo-controlled trials of darbepoetin alfa in the treatment of patients with chemotherapyinduced anemia. F Clin Oncol. 2009;27:2838-2847

18. Food and Drug Administration. Epoetin alfa label. http://www.accessdata. fda.gov/scripts/cder/drugsatfda/. Accessed May 21, 2013.

19. Moebus VJ, Jackisch C, Lueck HJ, et al. Intense dose-dense chemotherapy with epirubicin, paclitaxel and cyclophosphamide compared with conventionally scheduled chemotherapy in high-risk primary breast cancer: mature results of an AGO phase III study. F Clin Oncol. 2010;28:2874-2880.

20. Sobin LH, Wittekind Ch. International Union Against Cancer (UICC). TNM Classification of Malignant Tumors. 5th ed. New York: John Wiley \& Sons; 1997.

21. Henke M, Laszig R, Rube C, et al. Erythropoietin to treat head and neck cancer patients with anaemia undergoing radiotherapy: randomised, double-blind, placebo-controlled trial. Lancet. 2003;362:1255-1260

22. Hedenus M, Adriansson M, San Miguel J, et al. Efficacy and safety of darbepoetin alfa in anaemic patients with lymphoproliferative malignancies: a randomized, double-blind, placebo-controlled study. Br $\mathcal{F}$ Haematol. 2003;122:394-403

23. Wright JR, Ung YC, Julian JA, et al. Randomized, double-blind, placebocontrolled trial of erythropoietin in non-small-cell lung cancer with disease-related anemia. 7 Clin Oncol. 2007;25:1027-1032

24. Overgaard J, Hoff C, Sand Hansen H, et al. Randomized study of the importance of novel erythropoiesis stimulating protein (Aranesp) for the effect of radiotherapy in patients with primary squamous cell carcinoma of the head and neck (HNSCC): the Danish Head and Neck Cancer Group DAHANCA 10 rand. Eur 7 Cancer. 2007;5(Suppl):abstract 7.

25. Smith RE, Aapro MS, Ludwig H,et al. Darbepoetin alfa for the treatment of anemia in patients with active cancer not receiving chemotherapy or radiotherapy: results of a phase III, multicenter, randomized, double-blind, placebo-controlled study. 7 Clin Oncol. 2008;26:1040-1050

26. Untch M, Fasching PA, Konecny GE, et al. PREPARE trial: a randomized phase III trial comparing preoperative, dose-dense, dose-intensified chemotherapy with epirubicin, paclitaxel and CMF versus a standard dosed epirubicin/cyclophosphamide followed by paclitaxel \pm darbepoetin alfa in primary breast cancer-results at the time of surgery. Ann Oncol. 2011;22:1988-1998.

27. Thomas G, Ali S, Hoebers FJ, et al. Phase III trial to evaluate the efficacy of maintaining hemoglobin levels above $12.0 \mathrm{~g} / \mathrm{dL}$ with erythropoietin vs above $10.0 \mathrm{~g} / \mathrm{dL}$ without erythropoietin in anemic patients receiving concurrent radiation and cisplatin for cervical cancer. Gynecol Oncol 2008;108:317-325.

28. Bohlius J, Schmidlin K, Brillant C, et al. Recombinant human erythropoiesis-stimulating agents and mortality in patients with cancer: a metaanalysis of randomised trials. Lancet 2009;374:28.

29. Nitz U, Gluz O, Oberhoff C, et al. Adjuvant chemotherapy with or without darbepoetin alpha in node-positive breast cancer: survival and quality of life analysis from the prospective randomized WSG ARA Plus trial. Cancer Res. 2011;71(24 suppl):143s.

30. Swain SM, Tang G, Geyer CE Jr, et al. NSABP B-38: definitive analysis of a randomized adjuvant trial comparing dose-dense (DD) $\mathrm{AC} \rightarrow$ paclitaxel (P) plus gemcitabine $(\mathrm{G})$ with $\mathrm{DD} \mathrm{AC} \rightarrow \mathrm{P}$ and with docetaxel, doxorubicin, and cyclophosphamide (TAC) in women with operable, node-positive breast cancer. F Clin Oncol. 2012; 30(Suppl): abstract LBA1000.

31. Untch M, von Minckwitz G, Konecny GE, et al. PREPARE trial: a randomized phase III trial comparing preoperative, dose-dense, dose-intensified chemotherapy with epirubicin, paclitaxel, and CMF versus a standard dosed epirubicin/cyclophosphamide followed by paclitaxel with or without darbepoetin alfa in primary breast cancer-outcome on prognosis. Ann Oncol. 2011;22:1999-2006.

32. Aapro M, Jelkmann W, Constantinescu SN, et al. Effects of erythropoietin receptors and erythropoiesis-stimulating agents on disease progression in cancer. Br 7 Cancer. 2012;106:1249-1258.

33. Bennett CL, Lai SY, Henke M, et al. Association between pharmaceutical support and basic science research on erythropoiesis-stimulating agents. Arch Intern Med. 2010;170:1490-1498.

34. Elliott S, Busse L, Bass MB, et al. Anti-Epo receptor antibodies do not predict Epo receptor expression. Blood 2006;107: 1892-1895.

35. Hedley BD, Allan AL, Xenocostas A. The role of erythropoietin and erythropoieses-stimulating agents in tumor progression. Clin Cancer Res. 2011;17:6373-6380.

36. Chavez-Macgregor M, Zhao H, Kroll M, et al. Risk factors and incidence of thromboembolic events (TEEs) in older men and women with breast cancer. Ann Oncol. 2011;22:2394-2402.

37. Aapro MS, Link H. September 2007 update on EORTC guidelines and anemia management with erythropoiesis-stimulating agents. Oncologist 2008;13(Suppl):33-36.

38. Schrijvers D, De Samblanx H, Roila F; on behalf of the ESMO Guidelines Working Group. Erythropoiesis-stimulating agents in the treatment of anaemia in cancer patients: ESMO clinical practice guidelines for use. Ann Oncol. 2010;21(Suppl 5): v244-v247.

\section{Funding}

This study was supported by Bristol-Myers Squibb, Amgen, Pharmacia, and Johnson \& Johnson. A drug supply of epoetin alfa was provided by Johnson \& Johnson.

\section{Notes}

These funding sources had no influence on the design of the study, data collection, interpretation of data, and writing, or decision to submit the manuscript.

We thank all physicians and associates of the Arbeitsgemeinschaft Gynäkologische Onkologie (AGO) Breast Study Group. We also thank all trial patients for their invaluable contribution. We thank Bristol-Myers Squibb, Amgen, Pharmacia, and Johnson \& Johnson for supporting the study. We also thank Christina Weißmüller for her help in coordinating the study.

Presented in part at the 43rd Annual Meeting of the American Society of Clinical Oncology (ASCO), June 1-5, 2007, Chicago.

Affiliations of authors: Department of Gynecology and Obstetrics, Klinikum Frankfurt Höchst, Frankfurt, Germany (VM); Department of Gynecology and Obstetrics, Klinikum Offenbach $\mathrm{GmbH}$, Offenbach, Germany (CJ); National Center for Tumor Diseases, University of Heidelberg, Heidelberg, Germany (AS); Department of Gynecology and Obstetrics, University of Duesseldorf, Duesseldorf, Germany (JH); Gynäkologisch-Onkologische Praxis Hannover, Hannover, Germany (HJL); Department of Gynecology and Gynecologic Oncology, KEM Hussenstift, Essen, Germany (AdB); Department of Gynecology, University of Halle, Halle (Saale), Germany (CT); Medizinisches Zentrum BonnFriedensplatz, Bonn, Germany (CK); Department of Gynecology and Obstetrics, University of Bonn, Bonn, Germany (WK); Brustzentrum Niederrhein, Ev. Krankenhaus Bethesda, Mönchengladbach, Germany (UN); Department of Gynecology and Obstetrics, University of Jena, Jena, Germany (IBR); WiSP Research Institute, Langenfeld, Germany (AH); Department of Gynecology and Obstetrics, University of UIm, Ulm, Germany (RK); Department of Gynecology and Obstetrics, Helios Klinikum Berlin-Buch, Berlin, Germany (MU). 